Pacific Journal of Mathematics

MONOTONE OPERATORS AND NONLINEAR BIHARMONIC 


\title{
MONOTONE OPERATORS AND NONLINEAR BIHARMONIC BOUNDARY VALUE PROBLEMS
}

\author{
D. R. DUNNINGER AND JoHN LOCKER
}

The nonlinear boundary value problem $L u+N u=0$ is considered, where $L$ is the biharmonic operator and $N$ is a nonlinear monotone operator. By factoring the operator $L$ as $T T^{*}$, where $T$ is the maximal operator associated with the Laplacian, the theory of monotone operators is utilized to obtain an existence and uniqueness theorem for the operator equation. Several examples are given to illustrate the applicability of the result.

1. Introduction. In some recent works, Gustafson and Sather [6, 7] and Cesari and Kannan [4] have utilized the theory of monotone operators in order to prove the existence of solutions of the nonlinear differential equation $L u+N u=0$. Here $L$ is a linear differential operator with domain in a Hilbert space $S$ and $N$ is a nonlinear monotone operator, either defined on all of $S$ as in [4] or only on a subspace of $S$ as in $[6,7]$. In this paper we consider a similar problem in which $N$ is defined on a subspace of $S$ and where the differential operator is the biharmonic operator. The techniques of this paper are a modification of those used in [6,7]. In particular, whereas Gustafson and Sather make essential use of the square root decomposition of a positive self-adjoint operator, we use the fact that the biharmonic operator has a factorization of the form $T T^{*}$ where $T$ is the maximal operator of the Laplacian.

Let $\Omega$ be a bounded domain in $R^{n}$. For simplicity we assume that the boundary $\partial \Omega$ of $\Omega$ is of class $C^{\infty}$, although this condition can be relaxed somewhat (see [1, p. 28]). We consider the boundary value problem

$$
\left\{\begin{aligned}
\Delta \Delta u+N u=0 & \text { in } \quad \Omega, \\
u=\frac{\partial u}{\partial n}=0 & \text { on } \quad \partial \Omega,
\end{aligned}\right.
$$

where $\Delta$ denotes the $n$-dimensional Laplacian, $N$ is a nonlinear monotone operator, and $\partial / \partial n$ denotes the derivative in the direction of the inner normal to $\partial \Omega$. Our objective is to establish an existence and uniqueness theorem for problem (1). 
Let $S$ be the real Hilbert space $L^{2}(\Omega)$ with the standard inner product $(u, v)$ and norm $\|u\|$. For each integer $m \geqq 0$ let $W^{m}(\Omega)$ denote the subspace of all functions $u \in S$ such that the distribution derivatives $D^{\alpha} u \in S$ for $|\alpha| \leqq m$. Here and throughout $x=\left(x_{1}, \cdots, x_{n}\right) \in R^{n}$, $D^{\alpha}=D_{1}^{\alpha_{1}} \cdots D_{n}^{\alpha_{n}}$ where $D_{j}=\partial / \partial x_{j}, \alpha=\left(\alpha_{1}, \cdots, \alpha_{n}\right)$ is an $n$-tuple of nonnegative integers, and the order of $D^{\alpha}$ is $|\alpha|=\sum_{i=1}^{n} \alpha_{i}$. The space $W^{m}(\Omega)$ is a Hilbert space under the inner product and norm

$$
(u, v)_{m}=\sum_{|\alpha| \leqq m}\left(D^{\alpha} u, D^{\alpha} v\right), \quad\|u\|_{m}=(u, u)_{m}^{1 / 2}
$$

We refer to this structure for $W^{m}(\Omega)$ as its Sobolev structure. Let $W_{0}^{m}(\Omega)$ denote the closure in $W^{m}(\Omega)$, under the norm $\|\cdot\|_{m}$, of the subspace $C_{0}^{\infty}(\Omega)$ of infinitely differentiable functions with compact support in $\Omega$.

Let $L$ be the differential operator in $S$ defined by

$$
\mathscr{D}(L)=W^{4}(\Omega) \cap W_{0}^{2}(\Omega), \quad L u=\Delta \Delta u .
$$

In operator form equation (1) can be rewritten as

$$
L u+N u=0,
$$

where $N$ is a monotone operator with domain and range in $S$.

As has been pointed out by Kato [8], many operators of mathematical physics have the form $T T^{*}$. However, to the best knowledge of the authors, this decomposition has not been carried out for the biharmonic operator within the framework of Sobolev spaces. Consequently, in $\$ 2$ we discuss the structure of the linear operator $L$ and obtain the desired decomposition. In $\$ 3$ we present the nonlinear theory and prove our main existence and uniqueness theorem. We illustrate our main result in $\$ 4$ for a large class of nonlinear boundary value problems of type (1), and in $\$ 5$ analogous results are sketched for the corresponding Riquier problem [9], i.e., where $u=\Delta u=0$ on $\partial \Omega$.

2. Linear theory. We begin with a brief survey of the structure of the differential operator $L$. Important references in this regard are the two fundamental papers of Browder [1,2], to which we will be constantly referring. The essential features of our results in this section are summarized in Figure 1 at the end of this section.

The differential operator $L$ has the following properties:

(a) $\mathscr{D}(L)$ is a closed subspace in $W^{4}(\Omega)$ under its Sobolev structure. 
(b) $L$ is continuous from $\mathscr{D}(L)$ under the $W^{4}$-topology to $S$ under the $L^{2}$-topology.

(c) $L$ is a self-adjoint $\left(L=L^{*}\right)$ linear operator in $S$ (Theorem 16 $[1$, p. 76]).

(d) The null space $\mathcal{N}(L)=\{0\}$, and the range $\mathscr{R}(L)=S$.

From (d) it follows that

$$
H=L^{-1}
$$

exists. Clearly $H$ is a 1-1 linear operator from $S$ onto $\mathscr{D}(L)$. By the closed graph theorem $H$ is continuous from $S$ under the $L^{2}$-topology onto $\mathscr{D}(L)$ under the $W^{4}$-topology. Since $L=L^{*}$, we also have $H=H^{*}$.

Next we want to establish a decomposition of $L$ in the form $T T^{*}$. Let $T$ be the differential operator in $S$ defined by

$$
\begin{aligned}
\mathscr{D}(T)= & \left\{u \in S \mid \text { on every open subset } \Omega_{1} \subset \Omega\right. \text { with } \\
& \text { distance } d\left(\Omega_{1}, R^{n}-\Omega\right)>0, u \in W^{2}\left(\Omega_{1}\right), \\
& \Delta u \in S\}, T u=\Delta u .
\end{aligned}
$$

Then the adjoint operator $T^{*}$ is characterized by

$$
\mathscr{D}\left(T^{*}\right)=W_{0}^{2}(\Omega), \quad T^{*} u=\Delta u .
$$

The operators $T$ and $T^{*}$ are called the maximal and minimal operators, respectively, of the Laplacian $\Delta$ in $S$ (see [2, pp. 88-89 and Theorem 2.1, p. 129]). They are both closed operators in $S$.

These two operators have the following properties:

(a) $\mathcal{N}\left(T^{*}\right)=\{0\}$.

(b) $\mathcal{N}(T)$ is a closed subspace in $S$ under the $L^{2}$-topology.

(c) $\mathscr{R}\left(T^{*}\right)$ is a closed subspace in $S$ under the $L^{2}$-topology (see [2, Theorem 1.2, p. 97]).

(d) $\mathscr{R}(T)=S$.

Next, we introduce an inner product and norm for $\mathscr{D}\left(T^{*}\right)=W_{0}^{2}(\Omega)$ by

$$
(u, v)_{T^{*}}=(u, v)+\left(T^{*} u, T^{*} v\right), \quad u, v \in \mathscr{D}\left(T^{*}\right),
$$

and

$$
|u|_{T^{*}}=(u, u)_{T^{*}}^{1 / 2}, \quad u \in \mathscr{D}\left(T^{*}\right)
$$

Since $T^{*}$ is a closed operator in $S, \mathscr{D}\left(T^{*}\right)$ is a Hilbert space under this structure and $T^{*}$ is continuous from this Hilbert space to $\mathscr{R}\left(T^{*}\right)$ under the $L^{2}$-topology. Some elementary estimates together with Schwarz's 
inequality yield the inequality $|u|_{T^{*}} \leqq c\|u\|_{2}$, where $c$ is a positive constant, and we conclude that $T^{*}$ is continuous from $\mathscr{D}\left(T^{*}\right)$ under the $W^{2}$-topology onto $\mathscr{R}\left(T^{*}\right)$ under the $L^{2}$-topology. By the closed graph theorem $\left(T^{*}\right)^{-1}$ is continuous from $\mathscr{R}\left(T^{*}\right)$ under the $L^{2}$-topology onto $\mathscr{D}\left(T^{*}\right)$ under the $W^{2}$-topology.

For the space $\mathscr{D}(T)$ we introduce the inner product and norm

$$
(u, v)_{T}=(u, v)+(T u, T v), \quad u, v \in \mathscr{D}(T),
$$

and

$$
|u|_{T}=(u, u)_{T}^{1 / 2}, \quad u \in \mathscr{D}(T) .
$$

As above $\mathscr{D}(T)$ is a Hilbert space under this structure, and $T$ is continuous from this Hilbert space to $\mathscr{R}(T)=S$ under the $L^{2}$ topology. From the definition of $T$ it is clear that $\mathcal{N}(T) \neq\{0\}$. Consequently, we consider the restriction of $T$ to the subspace $\mathscr{D}(T) \cap$ $\mathcal{N}(T)^{\perp}$, where the orthogonal complement is in the $L^{2}$ sense. It is easily seen that $\mathscr{D}(T) \cap \mathcal{N}(T)^{\perp}$ is a closed subspace in the above Hilbert space, and $T \mid \mathscr{D}(T) \cap \mathcal{N}(T)^{\perp}$ is a 1-1 linear operator from $\mathscr{D}(T) \cap$ $\mathcal{N}(T)^{\perp}$ onto $S$.

Let

$$
J=\left[T \mid \mathscr{D}(T) \cap \mathcal{N}(T)^{\perp}\right]^{-1} .
$$

Then $J$ is a 1-1 linear operator from $\mathscr{R}(T)=S$ onto $\mathscr{D}(T) \cap \mathcal{N}(T)^{\perp}$, and by the closed graph theorem $J$ is continuous from $S$ under the $L^{2}$-topology onto $\mathscr{D}(T) \cap \mathcal{N}(T)^{\perp}$ under the $|\cdot|_{T}$-topology. This implies that $J$ is continuous from $S$ under the $L^{2}$-topology onto $\mathscr{D}(T) \cap \mathcal{N}(T)^{\perp}$ under the $L^{2}$-topology. Now the adjoint $J^{*}$ exists as an operator between $\mathcal{N}(T)^{\perp}=\mathscr{R}\left(T^{*}\right)$ and $S$ under their $L^{2}$-structures, and it is easy to show that $J^{*}=\left(T^{*}\right)^{-1}$.

We now show that the operators $T$ and $T^{*}$ yield the desired factorization of $L$.

LEMMA 1. $L=T T^{*}$.

Proof. Consider the product operator $T T^{*}$, which is defined by

$$
\begin{aligned}
\mathscr{D}\left(T T^{*}\right)= & \left\{u \in \mathscr{D}\left(T^{*}\right) \mid T^{*} u \in \mathscr{D}(T)\right\} \\
= & \left\{u \in W_{0}^{2}(\Omega) \mid \text { on every open subset } \Omega_{1} \text { of } \Omega\right. \\
& \text { with } d\left(\Omega_{1}, R^{n}-\Omega\right)>0, \Delta u \in W^{2}\left(\Omega_{1}\right), \text { and } \\
& \Delta \Delta u \in S\}, T T^{*} u=\Delta \Delta u .
\end{aligned}
$$

Clearly $L \subset T T^{*}$. Hence, the lemma will be established if we can show that $\mathscr{D}\left(T T^{*}\right) \subset \mathscr{D}(L)$. Towards this end let $u \in \mathscr{D}\left(T T^{*}\right)$ and set $f=$ 
$T T^{*} u=\Delta \Delta u \in S$. Since $\mathscr{R}(L)=S$, there exists a $v \in \mathscr{D}(L)$ such that $L v=\Delta \Delta v=f$. Hence the difference $w=u-v \in \mathscr{D}\left(T T^{*}\right)$ and $T T^{*} w=0$, which implies that $T^{*} w \in \mathcal{N}(T)$. On the other hand, $T^{*} w \in \mathscr{R}\left(T^{*}\right) \mathcal{N}(T)^{\perp}$. Thus, $T^{*} w=0$ and $w \in \mathcal{N}\left(T^{*}\right)=\{0\}$, and we have that $u=v \in \mathscr{D}(L)$, completing the proof of the lemma.

Since $\mathscr{D}\left(T T^{*}\right) \subset \mathscr{D}\left(T^{*}\right)$, we can consider the restriction $T_{R}=$ $T^{*} \mid \mathscr{D}\left(T T^{*}\right)$. It is easily verified that $T_{R}$ is a $1-1$ linear operator from $\mathscr{D}\left(T T^{*}\right)$ onto $\mathscr{D}(T) \cap \mathcal{N}(T)^{\perp}$.

Finally, we are able to conclude that

$$
H=L^{-1}=J^{*} J
$$

The preceding observations are depicted in Figure 1 below. Note that all operators involved are $1-1$ and onto their respective spaces. Moreover, the topologies under which these operators are continuous are also indicated.

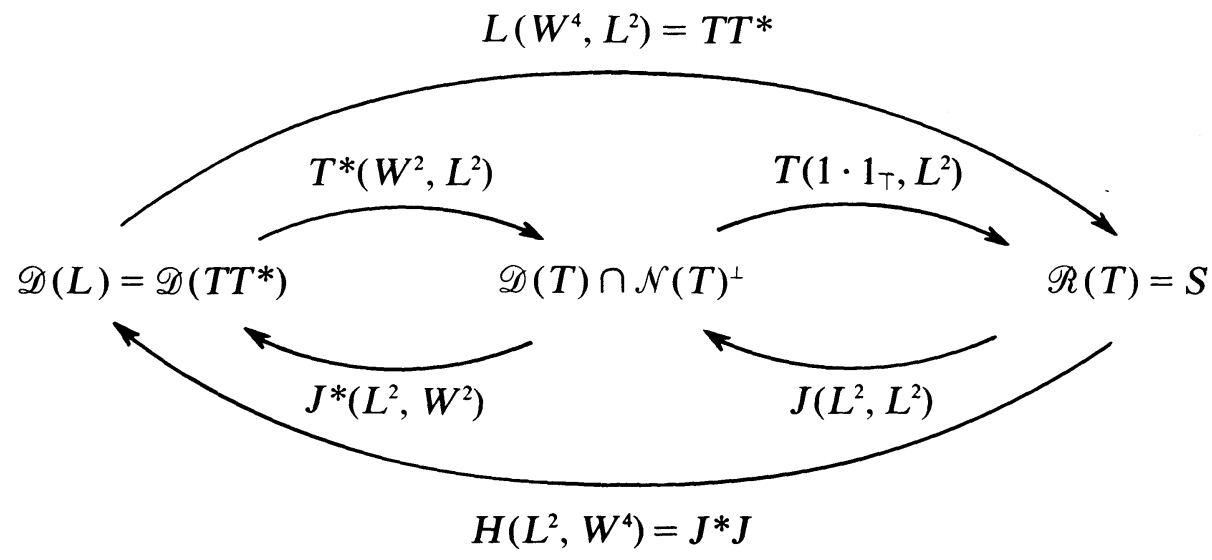

FiguRe 1

3. Existence and uniqueness. We now consider the boundary value problem

$$
L u+N u=0
$$

where $L$ is the linear operator in $\S 2$ and $N$ is a single-valued nonlinear operator with domain $\mathscr{D}(N) \supset \mathscr{D}\left(T^{*}\right)=W_{0}^{2}(\Omega)$ and range contained in $S$. Since $\mathscr{D}(L) \subset \mathscr{D}\left(T^{*}\right) \subset \mathscr{D}(N)$, equation (2) is defined for all $u \in$ $\mathscr{D}(L)$. 
Let $u \in \mathscr{D}(L)$ be a solution of (2). Applying $H$ to equation (2), we arrive at the so called "auxiliary equation"

$$
u+H N u=0 .
$$

If we set $v=T^{*} u$, then clearly $v \in \mathscr{D}(T) \cap \mathcal{N}(T)^{\perp} \subset \mathcal{N}(T)^{\perp}$ and $J^{*} v=$ $u$. Thus (3) becomes

$$
J^{*} v+J^{*} J N\left(J^{*} v\right)=0
$$

and since $J^{*}$ is $1-1$ we must have

$$
v+J N\left(J^{*} v\right)=0
$$

Equation (4) is an equation for $v$ in the Hilbert space $\mathcal{N}(T)^{\perp}$.

REMARK 1. In equation (4) the operators $J$ and $J *$ play the role of $K^{1 / 2}$ as defined in [7].

We now state our main result.

THEOREM 1. Let $\mathscr{D}(N) \supset W_{0}^{2}(\Omega)$ and assume that the operator $N$ from $\mathscr{D}(N)$ into $S$ satisfies the following conditions:

$\left(N_{1}\right) N$ is monotone on $W_{0}^{2}(\Omega)$, i.e., $(N u-N v, u-v) \geqq 0$ for all $u, v \in W_{0}^{2}(\Omega)$.

$\left(N_{2}\right) N$ is continuous from $W_{0}^{2}(\Omega)$ under the $W^{2}$-topology to $S$ under the $L^{2}$-topology.

Then the boundary value problem (2) has a unique solution $u \in$ $W^{4}(\Omega) \cap W_{0}^{2}(\Omega)$.

Proof. (Existence). Consider the operator $F$ mapping $\mathcal{N}(T)^{\perp}$ into $\mathcal{N}(T)^{\perp}$ which is defined by

$$
F v=v+J N\left(J^{*} v\right), \quad v \in \mathcal{N}(T)^{\perp} .
$$

From condition $\left(\mathrm{N}_{2}\right)$ and the continuity properties of $J$ and $J^{*}$, it follows that $F$ is continuous on $\mathcal{N}(T)^{\perp}$ under the $L^{2}$-topology. Also, for $u, v \in \mathcal{N}(T)^{\perp}$ we have

$$
\begin{aligned}
(F u-F v, u-v) & =\|u-v\|^{2}+\left(J N\left(J^{*} u\right)-J N\left(J^{*} v\right), u-v\right) \\
& =\|u-v\|^{2}+\left(N\left(J^{*} u\right)-N\left(J^{*} v\right), J^{*} u-J^{*} v\right) \\
& \geqq\|u-v\|^{2}
\end{aligned}
$$

where we have used condition $\left(N_{1}\right)$ in the last step. Thus, $F$ is strongly monotone on $\mathcal{N}(T)^{\perp}$. By the Browder-Minty theorem [3] and the 
strong monotonicity there exists a unique $v \in \mathcal{N}(T)^{\perp}$ such that $F v=0$, that is,

$$
v+J N\left(J^{*} v\right)=0 .
$$

Equation (5) implies that $v \in \mathscr{R}(J)=\mathscr{D}(T) \cap \mathcal{N}(T)^{\perp}$, and hence, we may define $u=J^{*} v \in \mathscr{D}(L)$. Then $T^{*} u=v$ and equation (5) becomes

$$
T^{*} u+J N u=0 .
$$

Applying $J^{*}$ yields

$$
u+J^{*} J N u=u+H N u=0,
$$

and applying $L$ yields

$$
L u+N u=0,
$$

which is the desired conclusion.

(Uniqueness). Suppose $u_{1}, u_{2} \in \mathscr{D}(L)$ are both solutions of (2). Let $v_{1}=T^{*} u_{1}, v_{2}=T^{*} u_{2}$. Clearly $v_{1}, v_{2} \in \mathcal{N}(T)^{\perp}$ and $F\left(v_{1}\right)=$ $F\left(v_{2}\right)=0$. Since $F(v)=0$ has a unique solution, we see that $v_{1}=v_{2}$, and hence, $u_{1}=J^{*} v_{1}=J^{*} v_{2}=u_{2}$. This completes the proof.

Remark 2. Condition $\left(N_{2}\right)$ can be replaced by the condition that $N$ be hemicontinuous, i. e., $N$ is continuous from each line segment in $\mathscr{D}(N)$ to the weak $L^{2}$-topology on $S$.

4. Examples. Let $n=1,2$, or 3 and let $\Omega$ be a bounded domain in $R^{n}$ with a $C^{\infty}$ boundary $\partial \Omega$. We consider the boundary value problem

$$
\left\{\begin{array}{ccc}
\Delta \Delta u+g(x, u)=f(x) & \text { in } & \Omega, \\
u=\frac{\partial u}{\partial n}=0 & \text { on } & \partial \Omega,
\end{array}\right.
$$

where $f \in S$ and $g$ is a continuous function from $\bar{\Omega} \times R$ to $R$ such that for each $x \in \Omega$ the function $g(x, \cdot)$ is monotone increasing on $R$.

Let $L$ be the differential operator defined by

$$
\mathscr{D}(L)=W^{4}(\Omega) \cap W_{0}^{2}(\Omega), \quad L u=\Delta \Delta u .
$$

By the Sobolev imbedding theorem it follows that under our restrictions on $n$ we have $W^{2}(\Omega) \subset C(\bar{\Omega})$ and

$$
\|u\|_{C(\bar{\Omega})} \leqq \text { const. }\|u\|_{2}
$$


for all $u \in W^{2}(\Omega)$. Let $N$ be the operator defined by

$$
\mathscr{D}(N)=W_{0}^{2}(\Omega), \quad N u(x)=g(x, u)-f(x) .
$$

Equation (6) becomes $L u+N u=0$.

We assert that $N$ has properties $\left(N_{1}\right)$ and $\left(N_{2}\right)$.

$\left(N_{1}\right)$ Monotone. For $u, v \in \mathscr{D}(N)$ we clearly have

$$
\begin{aligned}
{[N u(x)-N v(x)][u(x)-v(x)]=} & {[g(x, u(x))-g(x, v(x))] } \\
& \times[u(x)-v(x)] \geqq 0
\end{aligned}
$$

for all $x \in \Omega$, from which it follows that $N$ is monotone.

$\left(N_{2}\right)$ Continuity. Suppose $\left\|u_{i}-u\right\|_{2} \rightarrow 0$ as $i \rightarrow \infty$. Then in view of (7) the functions $u_{i}$ converge uniformly to $u$ on $\bar{\Omega}$, and hence, we can choose $M>0$ such that $\left|u_{i}(x)\right| \leqq M(i=1,2, \cdots)$ and $|u(x)| \leqq M$ for all $x \in \bar{\Omega}$. Since $g$ is uniformly continuous on $\bar{\Omega} \times[-M, M]$, it readily follows that the functions $g\left(x, u_{i}(x)\right)$ converge uniformly to $g(x, u(x))$ on $\bar{\Omega}$, which implies that $\left\|N u_{i}-N u\right\| \rightarrow 0$ as $i \rightarrow \infty$. Thus $N$ satisfies property $\left(\mathrm{N}_{2}\right)$.

By Theorem 1 equation (6) has a unique solution $u \in w^{4}(\Omega) \cap$ $W_{0}^{2}(\Omega)$. Moreover, by well-known regularity theorems [5], it is readily seen that if $f \in C^{2}(\bar{\Omega})$ and $g \in C^{2}(\bar{\Omega} \times R)$, then the solution $u$ is a classical solution in the sense that $u \in C^{4}(\Omega) \cap C^{1}(\bar{\Omega}), u$ satisfies $\Delta \Delta u+g(x, u)=f(x)$ at each point of $\Omega$, and $u=\partial u / \partial n=0$ on $\partial \Omega$.

As a specific example of equation (6) we can choose

$$
g(x, u)=a(x) u+b(x) u^{3}+c(x) e^{u},
$$

where $a(x), b(x)$, and $c(x)$ are nonnegative continuous real-valued functions on $\bar{\Omega}$.

5. The Riquier problem. Consider the boundary value problem

$$
\left\{\begin{array}{ccc}
\Delta \Delta u+N u=0 & \text { in } & \Omega, \\
u=\Delta u=0 & \text { on } & \partial \Omega .
\end{array}\right.
$$

The structure of the linear operator associated with this problem is considerably simpler than in the preceding case, and therefore the following discussion will be brief.

Let $T$ be the differential operator in $S$ defined by

$$
\mathscr{D}(T)=W^{2}(\Omega) \cap W_{0}^{1}(\Omega), \quad T u=\Delta u .
$$


As in $\$ 2$ it can be verified that $T$ is a $1-1$ continuous linear operator from $\mathscr{D}(T)$ under the $W^{2}$-topology onto $S$ under the $L^{2}$ topology. Hence, $J=T^{-1}$ is a $1-1$ continuous linear operator from $S$ under the $L^{2}$-topology onto $\mathscr{D}(T)$ under the $W^{2}$-topology. Moreover $T=T^{*}$ (see Theorem $16\left[1\right.$, p. 75]), and consequently, $J=J^{*}$.

The product operator $L=T T$ is the differential operator in $S$ given by

$$
\mathscr{D}(L)=\left\{u \in W^{4}(\Omega) \mid u \in W_{0}^{1}(\Omega), \quad \Delta u \in W_{0}^{1}(\Omega)\right\}, \quad L u=\Delta \Delta u .
$$

It is easily seen that $L$ is a $1-1$ self-adjoint linear operator in $S$ which is continuous from $\mathscr{D}(L)$ under the $W^{4}$-topology onto $S$ under the $L^{2}$-topology. The inverse $H=L^{-1}=J J$ is continuous from $S$ under the $L^{2}$-topology onto $\mathscr{D}(L)$ under the $W^{4}$-topology.

With $L$ given as above equation (8) can be written as

$$
L u+N u=0,
$$

where $N$ is a nonlinear operator with domain $\mathscr{D}(N) \supset \mathscr{D}(T)$ and range contained in $S$. If $N$ satisfies condition $\left(N_{1}\right)$ and $\left(N_{2}\right)$, then a proof similar to the proof of Theorem 1 yields the existence of a unique solution $u \in \mathscr{D}(L)$ of (8). Once again if $N u$ is sufficiently smooth, then the solution $u$ is a classical solution in the sense that $u \in C^{4}(\Omega) \cap$ $C^{2}(\bar{\Omega}), u$ satisfies $\Delta \Delta u+N u=0$ at each point of $\Omega$, and $u=\Delta u=0$ on $\partial \Omega$.

REMARK 3. In this last problem we have actually factored $L$ by finding its square root $T$. This method of attack has been carried out quite successfully in connection with other partial differential operators in the recent works of Gustafson and Sather $[6,7]$.

REMARK 4. Clearly the techniques of this paper can be extended to higher order elliptic problems in which the operator $L$ admits a factoring of the form $T T^{*}$. In particular, we cite the nonlinear polyharmonic problem:

$$
\left\{\begin{array}{cc}
\Delta^{2 k} u+N u=0 & \text { in } \quad \Omega, \\
\frac{\partial^{j}}{\partial n^{j}}=0 & \text { on } \quad \partial \Omega,
\end{array}\right.
$$

where $j=0,1, \cdots, 2 k-1$. 


\section{REFERENCES}

1. F. E. Browder, On the spectral theory of elliptic differential operators. I, Math. Ann., 142 (1961), 22-130.

2. - Functional analysis and partial differential equations. II, Math. Ann., 145 (1962), $81-226$.

3. - Nonlinear maximal monotone operators in Banach space, Math. Ann., 175 (1968), 89-113.

4. L. Cesari and R. Kannan, Functional analysis and nonlinear differential equations, Bull. Amer. Math. Soc., 79 (1973), 1216-1219.

5. A. Friedman, Partial Differential Equations, Holt, Rinehart and Winston, New York, 1969.

6. K. Gustafson and D. Sather, A branching analysis of the Hartree equation, Rend. Mat., 4 (1971), 723-734.

7. Large nonlinearities and monotonicity, Arch. Rational Mech. Anal., 48 (1972), 109-122.

8. T. Kato, On some approximate methods concerning the operators $T^{*} T$, Math. Ann., 126 (1953), 253-262.

9. M. Nicolesco, Les fonctions polyharmoniques, Actualités Sci. Ind., No. 331, Hermann, Paris, 1936.

Received June 10, 1974.

Michigan State University

AND

Colorado State University 
D. E. Bennett, Strongly unicoherent continua ............................. 1

Walter R. Bloom, Sets of p-spectral synthesis ................................ 7

R. T. Bumby and D. E. Dobbs, Amitsur cohomology of quadratic extensions: Formulas and number-theoretic examples ................. 21

W. W. Comfort, Compactness-like properties for generalized weak topological sums

D. R. Dunninger and J. Locker, Monotone operators and nonlinear biharmonic boundary value problems ...

T. S. Erickson, W. S. Martindale, 3rd and J. M. Osborn, Prime nonassociative algebras

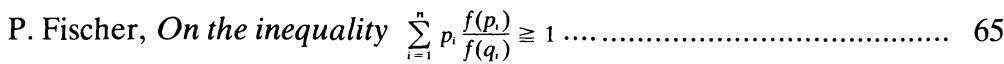

G. Fox and P. Morales, Compact subsets of a Tychonoff set ............... 75

R. Gilmer and J. F. Hoffmann, A characterization of Prüfer domains in terms of polynomials ......................................................... 81

L. C. Glaser, On tame Cantor sets in spheres having the same projection in each direction ......................................................... 87

Z. Goseki, On semigroups in which $X=X Y X=X Z X$ if and only if

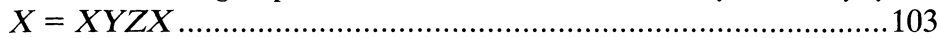

E. Grosswald, Rational valued series of exponentials and divisor

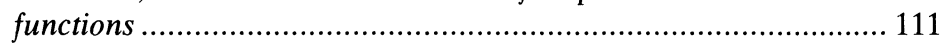

D. Handelman, Strongly semiprime rings ...................................... 115

J. N. Henry and D. C. Taylor, The $\bar{\beta}$ topology for $w^{*}$-algebras ............. 123

M. J. Hodel, Enumeration of weighted p-line arrays .......................... 141

S. K. Jain and S. Singh, Rings with quasiprojective left ideals .............. 169

S. Jeyaratnam, The diophantine equation $Y(Y+m)(Y+2 m) \times$

$$
(Y+3 m)=2 X(X+m)(X+2 m)(X+3 m) \ldots \ldots \ldots \ldots \ldots \ldots \ldots . . .183
$$

$\mathrm{R}$. Kane, On loop spaces without $p$ torsion .........................................189

Alvin J. Kay, Nonlinear integral equations and product integrals ..........203

A. S. Kechris, Countable ordinals and the analytic hierarchy, I ...........223

Ka-Sing Lau, A representation theorem for isometries of $C(X, E) \ldots \ldots . .229$

I. Madsen, On the action of the Dyer-Lashof algebra in $H_{*}(G)$..........235

R. C. Metzler, Positive linear functions, integration, and Choquet's theorem ........................................................................................ 277

A. Nobile, Some properties of the Nash blowing-up ............................297

G. E. Petersen and G. V. Welland, Plessner's theorem for Riesz conjugates 


\section{Pacific Journal of Mathematics}

\section{Vol. 60, No. $1 \quad$ September, 1975}

Donald Earl Bennett, Strongly unicoherent continua ................ 1

Walter Russell Bloom, Sets of p-spectral synthesis ................ 7

Richard Thomas Bumby and David Earl Dobbs, Amitsur cohomology of

quadratic extensions: formulas and number-theoretic examples .......

W. Wistar (William) Comfort, Compactness-like properties for generalized

weak topological sums ...................................

Dennis Robert Dunninger and John Stewart Locker, Monotone operators

and nonlinear biharmonic boundary value problems ..............

Theodore Erickson, Wallace Smith Martindale, III and J. Marshall Osborn,

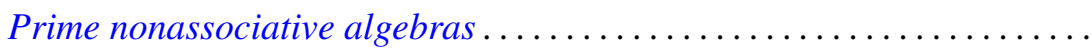

Pál Fischer, On the inequality $\sum_{i=0}^{n}\left[f\left(p_{i}\right) / f\left(q_{i}\right)\right] p_{i} \geq i \ldots \ldots \ldots \ldots \ldots$

Geoffrey Fox and Pedro Morales, Compact subsets of a Tychonoff set.......

Robert William Gilmer, Jr. and Joseph F. Hoffmann, A characterization of

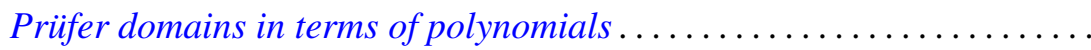

Leslie C. Glaser, On tame Cantor sets in spheres having the same projection

in each direction . ...................................

Zensiro Goseki, On semigroups in which $x=x y x=x z x$ if and only if

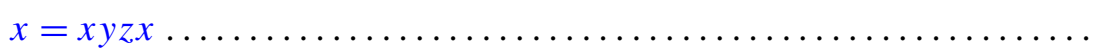

Emil Grosswald, Rational valued series of exponentials and divisor

functions.

David E. Handelman, Strongly semiprime rings

Jackson Neal Henry and Donald Curtis Taylor, The $\bar{\beta}$ topology for

$W^{*}$-algebras

Margaret Jones Hodel, Enumeration of weighted p-line arrays ...

Surender Kumar Jain and Surjeet Singh, Rings with quasi-projective left

ideals.

S. Jeyaratnam, The Diophantine equation

$$
Y(Y+m)(Y+2 m)(Y+3 m)=2 X(X+m)(X+2 m)(X+3 m) \ldots
$$

Richard Michael Kane, On loop spaces without $p$ torsion

Alvin John Kay, Nonlinear integral equations and product integrals ...

Alexander S. Kechris, Countable ordinals and the analytical hierarchy.

$I$.

Ka-Sing Lau, A representation theorem for isometries of $C(X, E)$

Ib Henning Madsen, On the action of the Dyer-Lashof algebra in $H_{*}(G)$

Richard C. Metzler, Positive linear functions, integration, and Choquet's

theorem.

Augusto Nobile, Some properties of the Nash blowing-up

Gerald E. Peterson and Grant Welland, Plessner's theorem for Riesz. 